\title{
LA PRECISIÓN DEL VOCABULARIO EN NIÑOS DEL GRADO PREESCOLAR
}

\section{THE PRECISION OF VOCABULARY IN PRESCHOOL CHILDREN}

\author{
Aymet Daniuska Torres - Montero 1 \\ Yannmila Hidalgo - Rosabal 2 \\ Miguel Alexis Casanova - Rosales 3
}

1.Cubana. Universidad de Granma. Sede Blas Roca Calderío, Email: adtorresm@estudiantes.udg.co.cu, Cuba.

2.Cubana. profesora titular, Universidad de Granma. Sede Blas Roca Calderío, Email:yhidalgorosabal@udg.co.cu, Cuba.

3.Cubano. Facultad de ciencias médicas de Granma, Celia Sánchez Manduley, mcasanovar@feu.grm.sld.cu, Cuba.

\begin{tabular}{|l|l} 
Recibido: 30.03 .19 & Aprobado: 15.07.19 \\
\hline
\end{tabular}

DOI:10.15517/isucr.v20i41.38809

\section{Resumen}

El presente trabajo se dirige a resolver las insuficiencias que se presentan en el desarrollo vocabulario en los niños del grado preescolar. Para darle solución a esta problemática se elaboraran adivinanzas infantiles que permiten la asimilación del significado de las palabras y con ello la precisión de su vocabulario. Las adivinanzas infantiles propuestas se establecen en tres contenidos: Animales, plantas, frutas y vegetales. En esta investigación la población está constituida por veintisiete niños del sexto año de vida de la escuela: Orestes Gutiérrez; se toma una muestra aleatoria de 14 niños, que representa el criterio de selección de forma aleatoria lo que constituyen el cincuenta y dos por ciento de la población. Por otro lado, se puede afirmar que las adivinanzas son dichos populares, juegos infantiles de ingenio que tienen como meta entretener y divertir a los niños contribuyendo al mismo tiempo a su aprendizaje, y a la enseñanza de un nuevo vocabulario. Por lo que la autora considera que el tema es de gran actualidad e importancia científica pues a partir de la propuesta se desarrolla la habilidad de observar y con ello los niños aprenden a centrarse en los detalles más importantes, a comparar e imaginar lo que posibilita que de forma lúdica los niños comprendan y amplíen el significado de los vocablos. Además de ser novedoso en cuanto a la muestra de una propuesta poco común y poco utilizada en las educadoras, para dirigir el proceso educativo como lo son dichas adivinanzas.

Palabras Claves: Niño; preescolar; vocabulario; adivinanzas infantiles; 


\section{Summary}

The present work is directed to solve the insufficiencies that appear in the vocabulary development in the children of the preschool level. To solve this problem, children's riddles will be developed that allow the assimilation of the meaning of the words and with it the precision of their vocabulary. The proposed children's riddles are established in three contents: Animals, plants, fruits and vegetables. In this investigation the population is constituted by twenty-seven children of the sixth year of life of the school: Orestes Gutierrez; a random sample of 14 children is taken, which represents the random selection criterion, which constitutes fifty-two percent of the population. On the other hand, it can be said that riddles are popular sayings, children's games of ingenuity that aim to entertain and entertain children while contributing to their learning, and teaching a new vocabulary. For what the author considers that the topic is very topical and scientific importance because from the proposal develops the ability to observe and with it the children learn to focus on the most important details, to compare and imagine what makes possible In a playful way, children understand and extend the meaning of the words. In addition to being innovative in terms of the sample of an unusual proposal and little used in educators, to direct the educational process as such riddles are.

Key Words: Child; preschool; vocabulary; children's riddles;

\section{Introducción}

La comunicación humana se realiza fundamentalmente con la ayuda del lenguaje verbal, que constituye, una forma peculiar de conocimiento de los objetos y fenómenos de la realidad, un reflejo de esta que se propicia por medio de la lengua natal, y que constituye a su vez, la principal vía de comunicación entre los seres humanos, mediante la cual entran en relación con sus semejantes, para coordinar acciones mutuas, intercambiar ideas e influirse entre sí.

De ahí que la lengua materna se asimila por imitación del habla del adulto y es indispensable que el modelo lingüístico de la educadora sea correcto de manera que constituya un ejemplo a imitar, esto es desde todo punto de vista: en la pronunciación de los sonidos de la lengua, corrección en el uso de vocablos y en la construcción gramatical así como en la fluidez, coherencia y claridad de las ideas.

Cuando un pequeño adquiere la palabra y aprende su significado, comienzaa combinarla de acuerdo con reglas gramaticales, y este proceso se produce de forma paulatina y ocurre en estrecha relación con el conocimiento del mundo natural y social.

En el tema se han destacado Loguinova, V. I. (1983) en la que se destaca una metodología para la adquisición de nuevos vocablos. Legaspi, A. (1997) con su organización didáctica para la adquisición del vocablo a partir de núcleos temáticos comunes relacionados con el conocimiento de la vida natural y social. En Cuba Chi L. (2004), investiga acerca de la adquisición del vocabulario Martínez, F (2004) investiga acerca de la importancia de la ejercitación de vocablos para el incremento de la amplitud del vocabulario y la eliminación o reducción de las dificultades fonéticas en la pronunciación, además propone los procedimientos metodológicos actuales para la adquisición de los vocablos en la infancia 
preescolar.

Otros autores son: Herrera, J. I (2008) propone un programa para estimular el lenguaje desde el componente léxico-semántico; Naranjo, M. (2008) ofrece una interesante propuesta práctica, desde la perspectiva de palabras que alcanzan un mayor nivel de generalización (palabras generalizadoras). Hidalgo, Y (2014) realiza una propuesta para estimular el vocabulario en el contexto familiar. También se realizan precisiones en por Mondeja, H 2017 para el trabajo con el vocabulario y se ofrecen aspectos teóricos que se perfeccionan partir del enfoque cognitivo comunicativo y sociocultural.

Se han estudiado además 5 tesis de maestría, realizadas en la provincia, relacionada con la estimulación del vocabulario sin embargo, la mayoría están relacionadas con la edad temprana y con la incorporación de poesía y cuentos para la presentación de vocablos. Existen pocas sugerencias para la precisión del significado de los vocablos.

Estas investigaciones se concentran en trabajar la adquisición del vocabulario de manera general sin precisar aspectos relacionados con la compresión y uso del significado de los vocablos lo que afecta el uso de vocablos en dependencia del contexto. Hablamos en este sentido del uso de vocablos para una conversación en una institución, con los adultos o con los niños lo que afecta demás la relación adulto- niño, niño- niño pues les resulta difícil la comunicación espontánea sin medie en ello la timidez pues escasamente preparamos a los niños para comunicarse en dependencia del contexto en el que actúan.

Es importante destacar que existen investigaciones relacionadas con los conceptos específicos y generales, Vigostki (), ya que existe una relación pensamiento- lenguaje y habla además de signos lingüísticos. Ese signo lingüístico es la asociación entre un concepto determinado y una imagen acústica, unión de tipo dialéctico entre pensamiento y sonido (inmaterial y material) y que comúnmente sabemos que en este caso estamos hablando de las palabras. Por ello el interés de incentivar la adquisición del significado de cada uno de los vocablos ya que por regularidad en la edad preescolar nos limitamos a presentar el vocablo para el niño aprenda el vocablo que denomina el objeto o fenómeno a partir de un modelo que le da la educadora.

Esto se pudo corroborar en las observaciones realizadas en el Seminternado Orestes Gutiérrez a las actividades programadas para vivenciar el desempeño de las educadoras y el desarrollo de los niños en cuanto al desarrollo del vocabulario se pudo constatar que los niños aún poseen dificultades para comprender el significado de los vocablos debido a que:

- Los niños aprenden de manera repetitiva las características de los objetos.

- El descubrimiento de las características, cualidades o funciones de los objetos es una práctica poco común entre las educadoras para precisar el significado.

- Las formas más utilizadas para la precisión de vocablos son la narración y la conversación.

La autora partiendo de estas insuficiencias determina el siguiente problema de investigación: ¿Cómo favorecer el desarrollo del vocabulario en los niños del grado preescolar? Para la solución del problema y alcanzar el propósito de la investigación se 
plantea como objetivo: Elaborar adivinanzas para la precisión del vocabulario en los niños del grado preescolar que favorezca su uso en diferentes situaciones comunicativas.

En esta investigación la población está constituida por veintisiete niños del sexto año de vida de la escuela: Orestes Gutiérrez; se toma una muestra aleatoria de 14 niños, que representa el criterio de selección de forma aleatoria lo que constituyen el cincuenta y dos por ciento de la población.

La novedad de la investigación se revela en el modo de dirigir el proceso educativo para la precisión del vocabulario a través de las adivinanzas que es un procedimiento poco utilizado para la ampliación del vocabulario de los niños.

El tema es de gran actualidad e importancia científica pues desarrollo a partir de la propuesta se desarrolla la habilidad de observar y con ello los niños aprenden a centrarla en los detalles más importantes, a comparar e imaginar lo que posibilita que de forma lúdica los niños comprendan y amplíen el significado de los vocablos.

\section{Desarrollo}

Dentro de los fundamentos teóricos sistematizados se encuentra: El concepto de lenguaje visto desde el punto de vista Filosófico, Psicológico y lingüístico. Fundamentos desde el punto de vista psicológico y lingüístico en función de las características del desarrollo psicológico y de la comunicación en los niños. Aspectos relacionados con la actividad y fundamentalmente lo relacionado con el enfoque lúdico que es vital en la etapa.

Se abordan aspectos relacionados con la definición de vocabulario, significado, signos lingüísticos. Desde el punto de vista didáctico-metodológico las diferentes metodologías utilizadas para la adquisición del vocabulario. El enfoque cognitivo, comunicativo y sociocultural que establece el uso de los vocablos con diferentes intenciones comunicativas y en diferentes contextos. Los métodos para el desarrollo de la comunicación en esta etapa. Desde el punto de vista Pedagógico, los principios de la educación preescolar y sus particularidades (López, J y Siverio, A. M. 2005). Los principios y niveles para la asimilación de los vocablos. Importancia de la literatura infantil para el desarrollo de la comunicación de los niños y específicamente de las adivinanzas como parte de la literatura y como método para el desarrollo de la comunicación.

\section{Resultados iniciales}

En el diagnóstico inicial se pudo evidenciar a través de la observación a las actividades programadas no siempre las educadoras confeccionan medios de enseñanza para su actividad, no crea situaciones que les permita al niño, descubrir por si solos, las características de los objetos y no siempre tiene en cuenta las necesidades de los niños para precisar los vocablos, por lo cual se encontró en el indicador de medianamente preparada. 
Por otro lado en la guía de entrevista a la maestra se pudo constatar que está poco preparada, porque presenta dificultades a la hora de trabajar correctamente el contenido que se está trabajando, no lo desarrolla con la calidad requerida.

En la encuesta realizada a la misma maestra se pudo constatar que está medianamente preparada, puesto que presenta limitaciones a la hora de guiar y orientar tanto a los niños como a la familia en el hogar, el trabajo con el vocabulario

En las pruebas pedagógicas realizadas a los niños se pudo constatar que: en la número uno, los niños tienen dificultades a la hora de identificar algunas frutas, plantas y animales, esto se pudo constatar ya que al mostrar las láminas: la cesta, el naranjal, la arquita de Noe, animales salvajes, el palmar, así como el Framboyán, solamente cuatro niños supieron identificar con exactitud a la cebra en la lámina: animales salvajes porque la confundían con un caballo; ocho solamente pudieron identificar, al mostrar la lámina de la cesta, al anoncillo y dos confundieron el limón con una guayaba. Al mostrar la lámina del Framboyán solamente tres niños me la supieron identificar correctamente, y al mostrar la lámina de la Palma Real tres niños la confundieron con la de coco y un niño dijo que su fruto era de coco.

En la segunda prueba, solamente nueve niños, supieron agruparme en una sola palabra lo que representaba la lámina, los animales salvajes y ninguno supo identificarme, que el tronco donde estaba la serpiente no era un animal. Diez me supieron decir en la lámina de la cesta, que todas eran frutas.

Y por último en la tercera prueba le mostré una lámina donde aparecía un tigre y un gato, y dos niños me dijeron que los dos eran un gato porque los dos eran anaranjados, tenían bigotes y arañaban, por tanto, no me supieron identificar en que se diferenciaban, también le mostré una lámina en la que aparecían naranjas y limones y cuatro niños me dijeron que eran naranjas, uno me dijo que el sabía que era naranja porque en su casa su papá tiene sembrada una mata, y dos me dijeron que ellos les gusta y se las comen bastante y una no me supo responder, el resto supo decirme correctamente y algunos con dificultad me respondieron las diferencias entre ellas, en la lámina de las plantas que se les mostró no hubo dificultades.

\section{Resultados finales}

Se aplicó el diagnóstico Final, donde la maestra, tanto en la guía de observación, como en la guía de entrevista y en la aplicación de la encuesta, arrojó favorables resultados porque venció todas las limitaciones y dificultades que presentaba a la hora de dirigir y orientar el proceso educativo del vocabulario tanto en los niños como a la familia.

Por su parte los niños, también obtuvieron buenos resultados, porque al final todos ya sabían identificar correctamente los animales que al inicio tenían dificultad para identificar, así mismo con las plantas y las frutas, y aprendieron a identificarlos también por su palabra generalizadora. Se puede decir entonces que se obtuvo el ciento por ciento de la preparación. 
III). ADIVINANZAS INFANTILES PARA LA PRESICIÓN DEL VOCABULARIO EN LOS NIÑOS DEL GRADO PREESCOLAR.

\section{ANIMALES}

Tengo rallas en todos lados; mis orejas son muy pequeñas.

$Y$ corro veloz en la selva detrás de un rico venado.
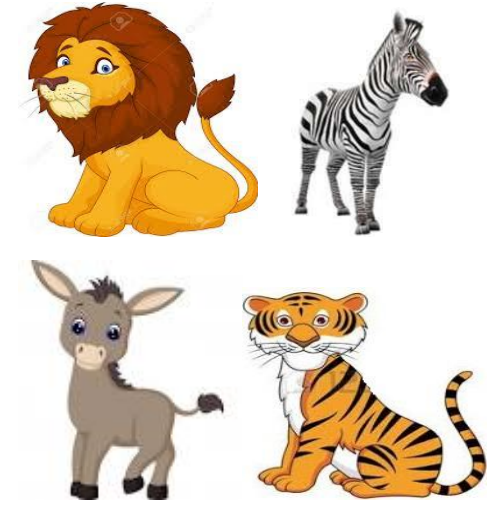

Soy un felino salvaje; de un negro color azabache, bigotes también yo tengo que me hacen rugir feroz.

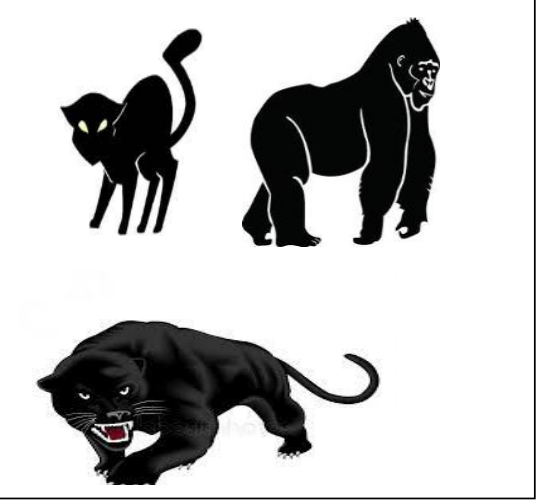

Mi cuerpo es amarillo,

cubierto de pequeñas plumas;

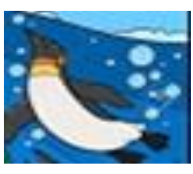

tengo un aplastado pico.

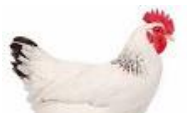

Y nado muy bien en el agua.

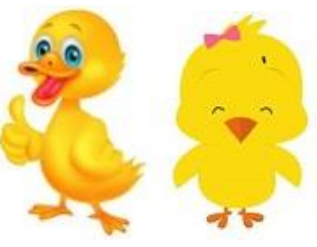

¿Quién soy?

Mi casa está en el mar

De gris o azul me sueles ver, y me temen las personas porque me las puedo comer.
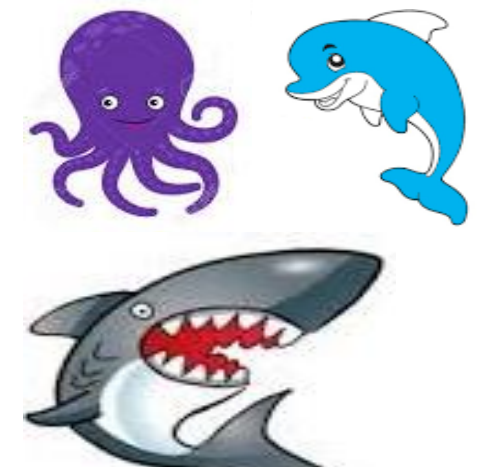


\section{PLANTAS}

Soy de verde penacho,

entre las plantas la más alta.

Con mis pencas formo el techo, y con mi cuerpo forro la casa.

Adivíname quién soy

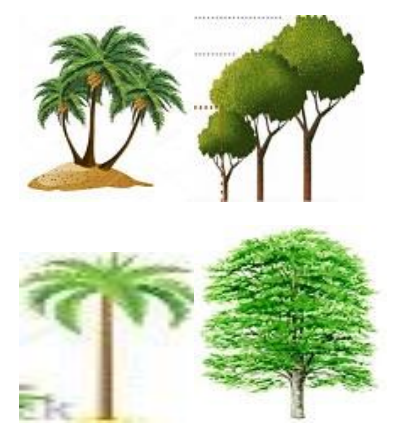

De muchos colores me encuentras; con hojas y espinas también.

A tu jardín realzo belleza y brindo fragancia a la vez.
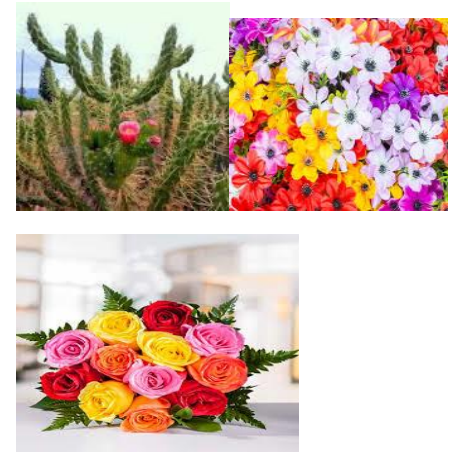


\section{FRUTAS}

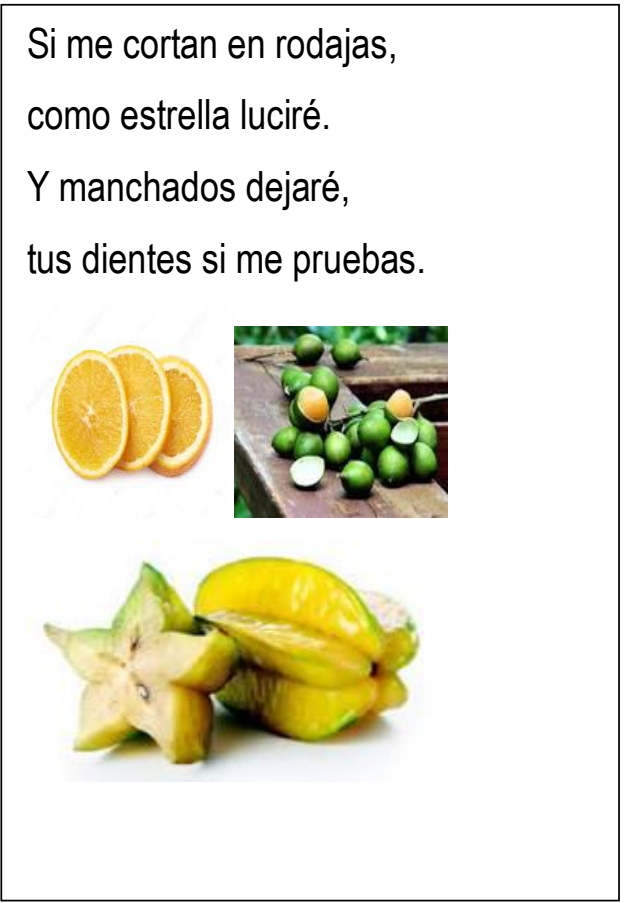

Es verde y grande por fuera, rojo es su interior con muchas, muchas semillas de oscuro y negro color ¿Qué es?

Sin ser princesa corona porto; ysin ser pez, yo tengo escamas.

Si me pelas verás muchos ojos que en mi masa se desparraman.
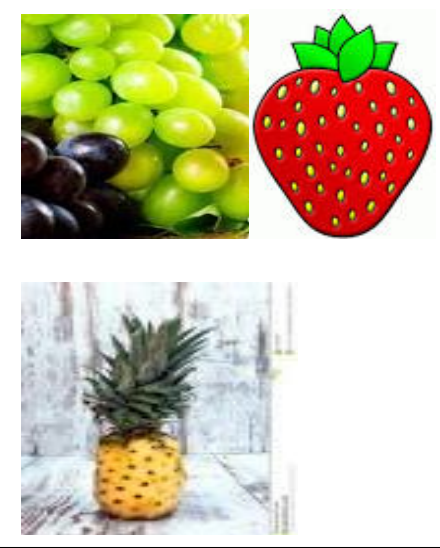


\section{CONCLUSIONES}

1. Los fundamentos teóricos del proceso educativo para los niños del grado preescolar avalan que el desarrollo del vocabulario es insuficientemente tratado en el contexto institucional. Los objetivos y contenidos están marcados por la poca motivación a que los niños descubran las características de los objetos, lo que limita la apropiación y ampliación de los significados y su uso en diferentes contextos y situaciones comunicativas.

2. El diagnóstico fáctico realizado evidencia insuficiencias en el desarrollo del vocabulario de los niños del grado preescolar y apuntan hacia el carácter reproductivo del significado de los vocablos en el proceso educativo que lo impulsa, lo que limita la adecuación contextual del significado de los vocablos para su uso activo.

3. La propuesta de Adivinanzas se estructuraron atendiendo a lo establecido en el programa las cuales responden a los contenidos de plantas, animales, y frutas.

4. Las adivinanzas infantiles propuestas contribuyeron a la preparación de los niños del grado preescolar de la escuela: Orestes Gutiérrez en cuanto a la precisión del vocabulario. 


\section{REFERENCIAS BIBLIOGRÁFICAS}

Acosta Padrón. R y Alfonso Hernández J. (s.a.). Didáctica interactiva. (En soporte electrónico).

Azcoaga, J.E. (2003) del lenguaje al pensamiento verbal. La Habana: Pueblo y Educación.

Báez García, M. (2006).Hacia una comunicación eficaz. La Habana: Pueblo y Educación.

Brumé, G. M. (1983).La lengua materna en el Círculo Infantil. La Habana: Pueblo y Educación.

Colectivo de autores. (2002). Compendio de Pedagogía. La Habana: Pueblo y Educación.

Entorno al Programa de Educación Preescolar. (1992) .La Habana: Pueblo y Educación.

Franco García, O. (2006b). Necesidad del enfoque lúdico en el proceso educativo de la Educación

García Batista, G. (Comp). (2002). Compendio de Pedagogía. La Habana: Pueblo y Educación.

Hidalgo Rosabal, Y. La orientación familiar para la precisión de vocablos a través de la formación de conjuntos en la edad preescolar (2013 noviembre). Revista Didascalia: Didáctica y Educación. Vol. IV, No 7. Monográfico Especial.

Legaspi, de A. (1997). Pedagogía Preescolar. La Habana: Pueblo y Educación.

Loguinova, V.I (1983). Formación del vocabulario. En el desarrollo del lenguaje en los niños de edad preescolar. pp. 100-127. La Habana: Pueblo y Educación.

Martínez Mendoza, F. (2004b). Lenguaje Oral. La Habana: Pueblo y Educación.

Orientaciones metodológicas para las educadoras sobre el programa de educación para los niños de 4to, 5to, 6to año de vida en el Círculo Infantil. (1981).La Habana: Pueblo y Educación.

Rodríguez Mondeja, H y López Lemus, V. Contribución al estudio de la Literatura para Preescolares. (1998) La Habana: Gente Nueva.

Rodríguez Mondeja, H y Rojas Bastard, A. (2010). La adquisición del lenguaje. Metodologías. La Habana: Pueblo y Educación.

Rodríguez Rivero, A.C y otros. (2010). Lecturas de Psicología preescolar. (En soporte electrónico).

Roméu Escobar A. (2007). El enfoque cognitivo, comunicativo y sociocultural en la enseñanza de la lengua y la literatura. La Habana: Pueblo y Educación.

Sojin, F.A. (1983). El desarrollo del lenguaje en los niños de edad preescolar. La Habana: Pueblo Educación.

Venguer, L (1978). Temas de Psicología. La Habana: Pueblo y Educación.

Vigotsky, L.V. Pensamiento y Lenguaje. (1998). La Habana: Pueblo y Educación. 